1. Van Etten, R. A. \& Shannon, K. M. Cancer Cell 6, 547-552 (2004).

2. Sanada, M. et al. Nature 460, 904-908 (2009).

3. Stephens, K et al. Blood 108, 1684-1689 (2006).

4. Fitzgibbon, J. et al. Cancer Res. 65, 9152-9154 (2005).

5. Kralovics, R., Guan, Y. \& Prchal, J. T. Exp. Hematol. 30, 229-236 (2002).

6. Dunbar, A. J. et al. Cancer Res. 68, 10349-10357 (2008)
7. Grand, F. H. et al. Blood 113, 6182-6192 (2009).

8. Loh, M. L. et al. Blood doi:10.1182/blood-2009-01-198416 (2009).

9. Schmidt, M. H. \& Dikic, I. Nature Rev. Mol. Cell Biol. 6, 907-918 (2005)

10. Rathinam, C. et al. Genes Dev. 22, 992-997 (2008).

11. Kotecha, N. et al. Cancer Cell 14, 335-343 (2008).

12. Mor, A. \& Philips, M. R. Annu. Rev. Immunol. 24, 771-800 (2006)

\title{
MICROBIAL GENETICS
}

\section{Love the one you're with}

\author{
Joseph Heitman
}

\section{Candida albicans is notorious as an opportunistic microbe that causes thrush and serious systemic disease. For geneticists, however, it offers continuing revelations into the wondrously varied sex lives of fungi.}

Sex facilitates evolution by recombining parental genomes and purging deleterious mutations. There are myriad forms, but they have three steps in common: meiosis, which halves the number of chromosomes per cell; gamete production; and cell-cell fusion. Sex in most animals involves two individuals of opposite sex, who produce distinct gametes (sperm and oocytes). In fungi, sex typically involves morphologically similar cells of opposite mating type ( $\mathbf{a}$ and $\alpha$ ) that reciprocally produce sex-stimulating pheromones. In other cases, fungi are self-fertile, and lone individuals can reproduce sexually. One well-known example of this is the model yeast Saccharomyces cerevisiae, in which a single cell divides, one of the resulting cells switches mating type, and mating yields a/a diploid cells, with a double complement of chromosomes, that no longer mate but undergo meiosis. On page 890 of this issue, Alby et al. ${ }^{1}$ report a conceptually similar but mechanistically distinct self-mating phenomenon in another yeast, the pathogenic Candida albicans.

Candida albicans resides in the human microbiota, for example in the gastrointestinal tract, and commonly causes infections. For more than a century it was thought to be strictly asexual. Then the mating-type gene locus was discovered, and most isolates were found to be a/a diploids that, like $S$. cerevisiae, are sterile ${ }^{2}$. Mutants with only one mating type $(\mathbf{a} / \mathbf{a}$ or $\alpha / \alpha)$ were a Opposite-sex mating in Candida albicans

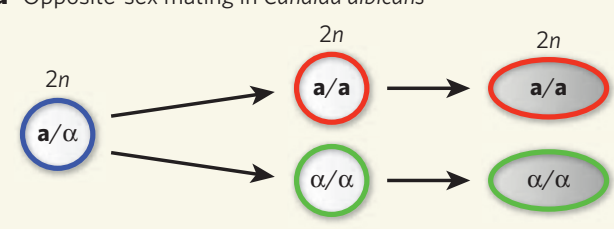

Loss of heterozygosity White-to-opaque switch

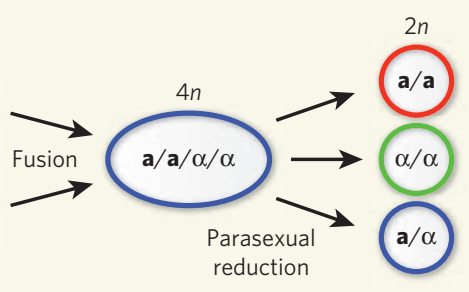

b Same-sex mating in Candida albicans

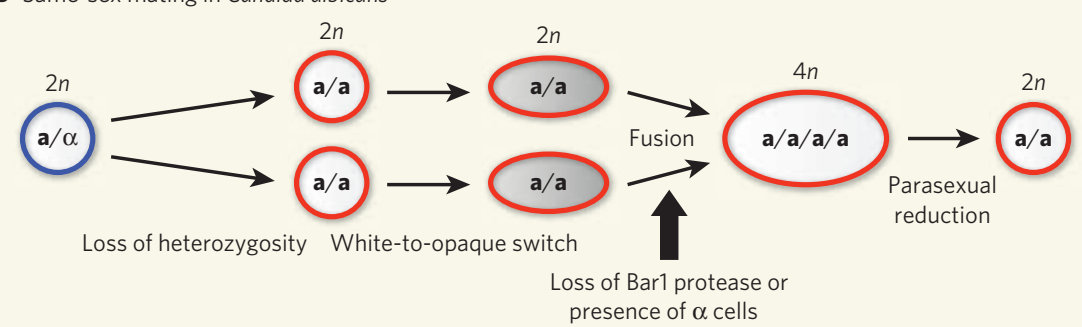

Figure 1 Opposite-sex and same-sex mating in Candida albicans. This fungus is diploid (2n): most strains are heterozygous, having both mating-type gene forms (a/a), and are sterile. a, Opposite-sex mating involves loss of heterozygosity, yielding a and $\alpha$ diploid cells that then switch from the white type to the opaque type specialized for mating. Cell-cell fusion yields tetraploid ( $4 n)$ cells in which parasexual chromosome reduction without recognized meiosis yields diploid progeny. $\mathbf{b}$, Same-sex mating again involves loss of heterozygosity and the white-opaque switch. As identified by Alby et al. ${ }^{1}$, the same-sex path can then be advanced in two ways, both of which involve production of pheromone of the opposite mating type that promotes fusion and results in tetraploid a cells. If a cells lack Bar1 protease, they do not destroy the $\alpha$-pheromone they produce; and in ménage-à-trois matings, even a few $\alpha$ cells can provide $\alpha$-pheromone.

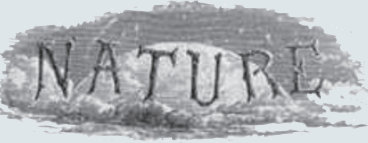

\section{YEARS AGO}

Most areas where there is a high prevalence of multiple sclerosis coincide in a highly suggestive fashion with areas where glaciation has played an important part in providing parent material for soils. However, the converse is certainly not true: all glacial soils cannot be correlated with areas where the prevalence of multiple sclerosis is high. Maps showing the distribution of multiple sclerotic cases in Northern Ireland, south-eastern Ontario, Sweden, and Denmark are alike in one respect-they all bear a remarkable resemblance to maps illustrating the distribution of boulders or geochemical anomalies in any map prepared for the purpose of searching in a glaciated area for buried ore bodies... One other point appears worthy of note: higher than 'normal' quantities of lead are known to occur in those rocks ... occurring in areas where the prevalence of multiple sclerosis is high ... It should also be noted that anomalous amounts of lead may, on occasion, be accompanied by anomalous amounts of some other elements such as silver, barium, magnesium, and fluorine. From Nature 15 August 1959.

\section{YEARS AGO}

It is a usual custom in pharmacological work to state the dosage of drugs as so much per kilogram of body-weight of animal or man, the subject of experiment or treatment. Prof. Benjamin Moore points out in the Biochemical Journal ... that this method of stating dosage is inaccurate, the dose of a drug for two individuals of different size, apart from peculiar idiosyncrasies, being proportional, not to their weights, but to their body surfaces, in other words, to the two-thirds powers of their weights. Thus an adult of $150 \mathrm{lb}$. weight cannot be given fifteen times the dose for an infant of $10 \mathrm{lb}$., but much more nearly a dose only six times as much. It may be that it is this principle which limits the value of some drugs. From Nature 12 August 1909. 
a Same-sex mating in Candida albicans

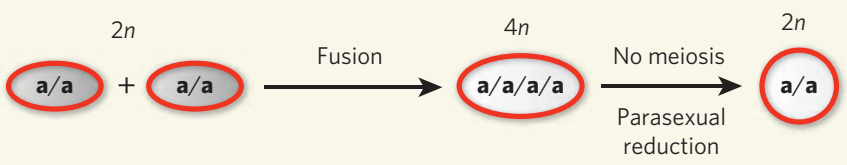

b Same-sex mating in Cryptococcus neoformans

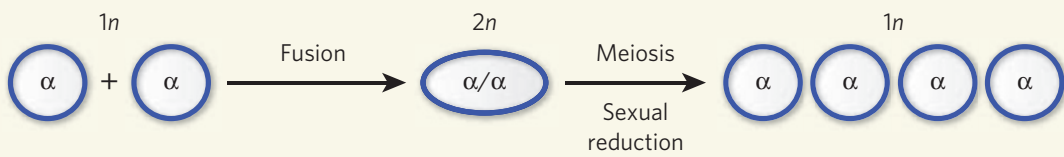

Figure 2 | Same-sex mating in two pathogenic fungi. Unisexual reproduction occurs in both Candida albicans (a) and Cryptococcus neoformans (b). These forms of self-fertility may result in population features (such as inbreeding and clonality) that are associated with pathogenesis. Given that two such evolutionarily divergent pathogenic fungi both undergo same-sex mating, this process is likely to be even more general among fungi. The two species are distinguished in that mating in C. albicans is parasexual and no spores are produced, whereas in C. neoformans meiosis results in the production of infectious spores.

found to mate in the laboratory or during infection $^{2}$. But the low frequency of mating raised the question of whether sex occurs in nature. This was resolved by various discoveries ${ }^{2-4}$, including the observation that mating is enhanced a million-fold when cells switch from 'white' to an 'opaque' type specialized for mating.

In opposite-sex mating in C. albicans (Fig. 1a), cell-cell fusion produces tetraploid cells that have four copies of each chromosome $(\mathbf{a} / \mathbf{a} / \alpha / \alpha)$. These tetraploids undergo a 'parasexual' phase, which restores the diploid state and allows limited genetic recombination but does not involve overt meiosis and does not produce spores. (The parasexual recombination that does occur requires Spo11 (ref. 6), an enzyme that acts as a recombinase during meiosis in other organisms. Thus, sex in C. albicans could involve cryptic meiosis or novel parasexual roles for Spo11.)

Alby et al. ${ }^{1}$ report the discovery of a facet of C. albicans reproduction in which cells mate with each other via same-sex mating (Fig. 1b), rather than with the opposite mating type. This observation arose from analysing C. albicans mutants lacking barrier protease, an enzyme that inactivates $\alpha$-pheromone. Barrier was discovered in S. cerevisiae as a factor that interferes with mate signalling when a cells producing barrier are interposed between a and $a$ cells ${ }^{7}$. Among other things, we now know that barrier is encoded by the BAR1 gene in a mating-type-specific manner, and that it acts on $\alpha$-pheromone, enabling a cells to choose mates producing the most pheromone.

Curiously, C. albicans a cells express genes encoding both a- and $\alpha$-pheromone. Alby et al. ${ }^{1}$ observed that a cell bar 1 mutants lacking barrier produce unusual wrinkled colonies, and that these colonies were responding to pheromone. Surprisingly, they also discovered that $\mathbf{a}-\mathbf{a}$ cell fusion can occur both between bar1 mutants and between wild-type and bar1-mutant cells. Crosses with mutant strains showed that same-sex mating occurs when $\alpha$-pheromone is produced by one a cell and is sensed by $\alpha$-pheromone receptors on both the producing and the partner a cell.

Alby et al. found that same-sex mating is less efficient than opposite-sex mating, but is readily detectable, and in some cases is appreciable. They also observed same-sex mating between wild-type a cells (producing Bar1) in ménage$\grave{a}$-trois matings in which an $\alpha$-cell minority provided $\alpha$-pheromone. Under these conditions, sufficient $\alpha$-pheromone may overwhelm Bar1 and trigger $\mathbf{a}-\mathbf{a}$ cell fusion. Same-sex mating yielded tetraploid cells $(\mathbf{a} / \mathbf{a} / \mathbf{a} / \mathbf{a})$ that could undergo parasexual chromosome loss to diploid progeny. Thus, C. albicans does not need both mating-type locus versions for the parasexual cycle, in contrast to their requirement for meiosis in S. cerevisiae.

Do opposite- and same-sex mating occur in nature? The original discovery of inefficient $C$. albicans $\mathbf{a}-a$ mating fuelled speculation that it could be a lab artefact: however, a complex cellular network has been maintained that supports efficient a- $\alpha$ mating. Populationgenetics studies and identification of matingstimulatory host conditions lend further support to the view that opposite-sex mating happens in nature ${ }^{2-4}$. That same-sex mating occurs most efficiently with mutant and not wild-type strains suggested that bar1 mutants might occur in the population. No natural barl mutants have been identified so far, however, and they might arise only transiently. Alternatively, host conditions or protease inhibitors produced by $C$. albicans ( $\alpha$-pheromone variants) might stimulate same-sex mating in nature. Even infrequent same-sex mating in ménage-à-trois matings might suffice for genetic exchange in skewed mating-type populations, such as commensal biofilms on host mucosal membranes.

Although it might seem odd that yeast would mate by both opposite-sex/outcrossing and same-sex/inbreeding, S. cerevisiae is again instructive. The natural population consists of isolates that switch mating type and self-mate, and others that cannot switch but are fertile with the opposite mating type. Thus, a well-defined species can evidently consist of individuals with different modes of sexual reproduction. It is striking, however, that two closely related yeasts (C. albicans and S. cerevisiae) evolved such distinct modes of self-fertility. The more evolutionarily divergent fission yeast, Schizosaccharomyces pombe, also switches mating type and self-mates, like S. cerevisiae, but independently evolved a machinery to do so. This diversity in forms of sexual reproduction illustrates the remarkable plasticity of fungi, which have evolved to exploit different environments.

A divergent pathogenic yeast, Cryptococcus neoformans, also reproduces unisexually ${ }^{8}$. This species has a well-defined $\mathbf{a}-\boldsymbol{\alpha}$ sexual cycle, and the fact that the natural population is largely a unisexual was puzzling. The discovery that a cells undergo same-sex mating, yielding diploid cells that complete meiosis and produce spores (Fig. 2), suggested another route by which diversity might be maintained ${ }^{8}$. Population-genetics studies provide evidence that same-sex mating occurs in nature, and implicate the process in an outbreak of Cryptococcus infection ${ }^{9,10}$. That same-sex mating arose convergently in two divergent pathogenic yeasts suggests that unisexual reproduction may be linked to microbial pathogenesis, and could be even more widespread than is currently appreciated.

Transitions in sexual reproduction between inbreeding and outbreeding also occur in plants and animals. In plants, mutations in self-incompatibility loci involved in pollen recognition evoke transitions from crosspollination to self-pollination ${ }^{11}$. One individual can thereby colonize different environmental niches, and become more cosmopolitan. Parthenogenesis - virgin birth - is restricted in mammals by genomic imprinting, but remarkable examples have been reported in Komodo dragons and sharks ${ }^{12,13}$. In these cases, otherwise sexual organisms reproduce under extreme circumstances, possibly enabling the colonization of islands or oceanic expanses by single individuals.

Joseph Heitman is in the Department of Molecular Genetics and Microbiology, Duke University Medical Center, Durham, North Carolina 27710, USA.

e-mail: heitm001@duke.edu

1. Alby, K., Schaefer, D. \& Bennett, R. J. Nature $460,890-893$ (2009).

2. Bennett, R. J. \& Johnson, A. D. Annu. Rev. Microbiol. 59 233-255 (2005).

3. Daniels, K. J. et al. EMBO J. 25, 2240-2252 (2006)

4. Huang, G. et al. Curr. Biol. 19, 330-334 (2009).

5. Bennett, R. J. \& Johnson, A. D. EMBO J. 22, 2505-2515 (2003)

6. Forche, A. et al. PLoS Biol. 6, e110 (2008).

7. Hicks, J. B. \& Herskowitz, I. Nature 260, 246-248 (1976).

8. Lin, X., Hull, C. M. \& Heitman, J. Nature 434, 1017-1021 (2005).

9. Fraser, J. A. et al. Nature 437, 1360-1364 (2005)

10. Lin, X. et al. PLoS Genet. 3, e186 (2007).

11. Tang, C. et al. Science 317, 1070-1072 (2007)

12. Watts, P. C. et al. Nature 444, 1021-1022 (2006).

13. Chapman, D. D. et al. Biol. Lett. 3, 425-427 (2007). 